\title{
A Fault-Tolerant Real-Time Scheduling Algorithm in Software Fault-Tolerant Module
}

\author{
Dong Liu ${ }^{1}$, Weiyan Xing ${ }^{2}$, Rui $\mathrm{Li}^{1}$, Chunyuan Zhang ${ }^{1}$, and Haiyan $\mathrm{Li}^{1}$ \\ ${ }^{1} 6$ yuan 7 dui, Department of Computer, National University of Defense Technology, \\ Changsha, Hunan 410073, China \\ windleaf1980@163.com \\ ${ }^{2}$ China Huayin Ordnance Test Center, Huayin 714200, China
}

\begin{abstract}
In software fault-tolerant module, the key issue that affects the performance of fault-tolerant scheduling algorithm is how to predict precisely whether a primary is executable. In order to improve the prediction precision, a new algorithm named DPA, Deep-Prediction based Algorithm, is put forward. DPA uses prediction-table to predict whether a primary can be completed before it is scheduled. Simulation results show that DPA provides more execution time for primaries and wastes less processor time than the wellknown similar algorithms.
\end{abstract}

Keywords: scheduling algorithm, software fault-tolerance, real-time system.

\section{Introduction}

In addition to the function constraints and time constraints, real-time systems in lifecritical applications should supply high reliability so that the systems would not halt in the presence of task failure. Therefore, various fault-tolerant modules were put forward to solve the problem. One of the widely used modules is software faulttolerant module, which uses uniprocessor scheduling algorithms to schedule two versions for each real-time task, namely primary and alternate [1] [2] [3].

In software fault-tolerant module, real-time system has a set of $n$ real-time periodic tasks $\tau=\left\{\tau_{1}, \tau_{2}, \ldots, \tau_{n}\right\} . \tau_{i}$ has a period $T_{i}$ and two independent versions of computation program: the primary $P_{i}$ and the alternate $A_{i} . P_{i}$ has a computation time $p_{i}, A_{i}$ has a computation time $a_{i}$, and, usually, $p_{i} \geq a_{i}$ for $1 \leq i \leq n$.

Let the planning cycle, $T$, be the least common multiple (LCM) of $T_{1}, T_{2}, \ldots, T_{n}$. We only need to consider all task invocations during the first planning cycle $[0, T]$. The primary and the alternate of the $j$ th job, $J_{i j}$, of $\tau_{i}$ are denoted by $P_{i j}$ and $A_{i j}$. We define $r_{i j}=(j-1) T_{i}$ to be the release time and $d_{i j}=j T_{i}$ be the deadline of $J_{i j}$.

Since $P_{i j}$ provides a better computation quality, we expect to complete as many primaries as possible while guaranteeing either the primary or the alternate of each task to be successfully completed by its deadline. If there are primaries pending for execution, alternate will not be scheduled until the latest possible time, called the notification time, denoted by $v_{i j}$. Therefore, we can say that $v_{i j}$ is the pre-deadline of $P_{i j}$. FP is denoted as the probability that the primary fails after its execution. 
[1] proposed BCE (Basic \& CAT \& EIT) algorithm to provide fault-tolerant scheme in software fault-tolerant module. BCE includes three sub-algorithms: Basic, CAT and EIT. Basic algorithm uses a fixed priority-driven preemptive scheduling scheme to pre-allocate time intervals to alternates as late as possible, and, at runtime, attempts to execute primaries before alternates. Since the prediction of an executable primary is the key issue that affects the scheduling performance, CAT (Check Available Time) algorithm is adopted to make prediction and abort the primary that has not sufficient time to finish. When processor becomes idle, EIT (Eliminate Idle Time) algorithm advances the scheduling of the alternate, which has the highest priority, to save time.

[2] and [3] enhanced BCE algorithm and introduced two algorithms: PKSA (Probing K-steps Algorithm) and CUBA (Changing Utilization-Based Algorithm). Both algorithms probe $K$ steps, which are the probing deepness of the algorithms, before the executions of primaries, so that they can prevent early failure of one primary from triggering failures in subsequent tasks' executions. In this way, the algorithms increase the percentage of successfully completed primaries compared to BCE. Generally, PKSA works better than CUBA [2].

On the base of BCE and PKSA [1] [2], this paper proposes Deep-Prediction Based Algorithm (DPA) in order to precisely predict whether a primary is executable.

\section{DPA Scheduling Algorithm}

In this paper, we use a time-table, named prediction-table (PT), to make prediction for $P_{i j}$, named host primary. PT stores the execution information of $P_{i j}$ and all its preemptive primaries $P_{m n}$ and alternates $A_{m n}$.

Theorem 1. Let the current time be $t, P_{i j}$ has been released, and the notification time of $A_{i j}$ is $v_{i j}$. If the total time that has been allocated for primaries (not including $P_{i j}$ ) and alternates during $\left[t, v_{i j}\right]$ is $I$, then the available time of $P_{i j}$ is $A T=v_{i j}-t-I$.

Proof. Since $P_{i j}$ must be completed by its deadline $v_{i j}$, the maximum available time for $P_{i j}$ to use will be $v_{i j}-t$. During $\left[t, v_{i j}\right]$, besides $P_{i j}$, there exist the primaries $P_{m n}$, which preempt $P_{i j}$, and the alternates, whose primaries have failed or been aborted. All of the primaries and the alternates preempt $P_{i j}$ in PT, and the total time occupied by these tasks is $I$. Consequently, the available time, denoted by $A T$, for $P_{i j}$ in PT will be $v_{i j}-t-I$. This is the relation we desired to prove.

The procedure of constructing PT for host primary $P_{i j}, \mathrm{PT}\left(P_{i j}\right)$, is described below:

Let $t$ denote the current time. $P_{i j}$ has been released.

(1) PT is initialized to be idle. Set the start-time and the end-time of PT to be $t$ and $v_{i j}$ respectively. Then, copy the time intervals that have been allocated to alternates by backward-RM scheduling algorithm [1] during [t, $\left.v_{i j}\right]$ to PT. An alternate-list, containing all the alternates in PT, is created, and the alternates in the list are sorted ascendingly in order of their notification time.

(2) Let $A_{m n}$ denote the first unchecked alternate, and $P_{m n}$ be its primary. If $P_{m n}$ has been aborted or failed, go to (2) to check the next alternate in alternate-list; otherwise, go to (3). If there is no unchecked alternate in alternate-list, go to (4). 
(3) Judge whether the available time in PT is sufficient for $P_{m n}$ using Theorem 1. If $P_{m n}$ can meet its pre-deadline, then append $P_{m n}$ 's time into PT and withdraw the time intervals occupied by $A_{m n}$. Otherwise, abort $P_{m n}$. Go to (2).

(4) Judge whether the available time in PT is sufficient for $P_{i j}$ using Theorem 1 . If $P_{i j}$ can meet its pre-deadline, append $P_{i j}$ 's time into PT, and return "successful". If $P_{i j}$ can not finish, return "failed".

The following algorithm describes DPA in detail.

\section{Procedure DPA( )}

(1) Set the current time, $t$, to be 0 ; set the current scheduling state, $S$, to be "not PT'.

(2) Use the backwards-RM algorithm to allocate time intervals for all alternates.

(3) The current time is $t$.

(3.1) If $S=$ "not PT", then:

(3.1.1) If an alternate arrives, execute the alternate. Go to (4);

(3.1.2) If the processor is idle, do EIT algorithm. Go to (3)..

(3.1.3) If a primary arrives, let $P_{i j}$ denote the primary whose alternate has the smallest notification time among all the released primaries. If $\operatorname{PT}\left(P_{i j}\right)=$ "successful", set $S$ to "PT"; otherwise, abort $P_{i j}$. Go to (3).

(3.2) If $S=$ "PT", let $P_{i j}$ denote the host primary. Then:

(3.2.1) If it is time to execute $P_{i j}$ in PT, schedule $P_{i j}$. If $P_{i j}$ is completed, set $S$ to "not PT". If there is no fault during $P_{i j}$ 's execution, remove $A_{i j}$; otherwise, reserve $A_{i j}$.

(3.2.2) If it is time to execute $P_{m n}\left(P_{m n} \neq P_{i j}\right)$ in PT, schedule $P_{m n}$. If $P_{m n}$ is completed and failure occurred during its execution, set $S$ to "not PT".

(3.2.3) it is time to execute $A_{m n}$ in PT, schedule $A_{m n}$.

(4) Set the next time, and go to (3).

In the next section, we will use simulations to demonstrate the efficiency of DPA compared to PKSA. (Since PKSA is better than CUBA [2], we only compare PKSA and DPA.)

\section{Simulation Results}

We use two metrics: PSP (Percent of time used by Successful Primaries) and $W$ (Wasted time units). PSP is the percentage of time used by successfully completed primaries. $W$ is the time wasted by executing inexecutable (not failed) primaries.

In the simulation, we set FP to be 0.05 and set the probing deepness of PKSA to be 4, 8 and 16. We simulated five tasks with different $T_{i}, p_{i}, a_{i}$ for 500 times. $T_{i}$ of each task was between 10 and 150 time units and $a_{i}$ was 0.1 times of $T_{i}$. By adjusting the proportion of primaries' computation time to their periods, we can control the utilization of primaries, $U_{P}$. We simulated 10000 time units in each simulation. Through this way, we got 500 results of PSP and $W$, respectively, and then the results were averaged. The averages are exactly the final results shown in Fig.1. 

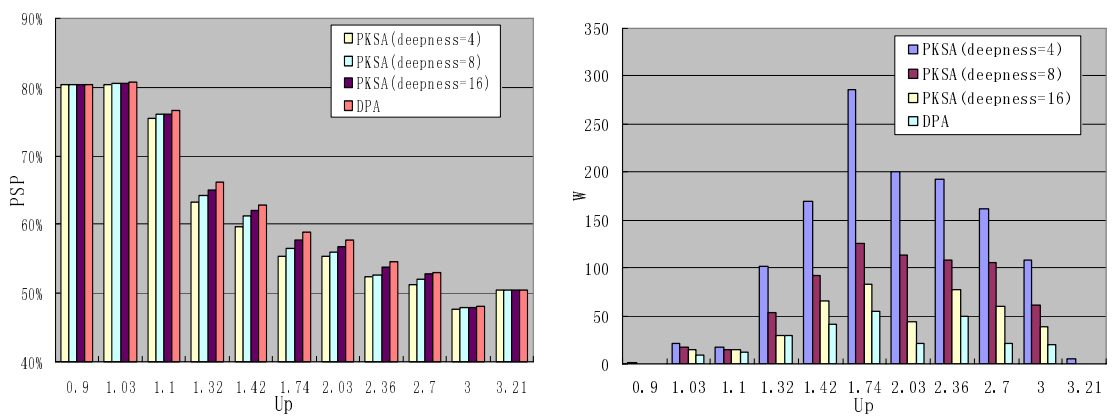

Fig. 1. Comparison between DPA and PKSA with different $U_{p}$

When $U_{P}<1.1$, primaries' computation time is short, and both algorithms can predict that most of primaries have sufficient available time to execute, so two algorithms get similar scheduling performances. When $1.1<U_{p}<3$, DPA wastes less processor time and provides more time to successful primaries compared to PKSA. When $U_{p}>3$, the performances of two algorithms get close again, since most primaries can not be completed before notification time.

\section{Conclusion}

In this paper, we proposed DPA algorithm for software fault-tolerant module. The algorithm constructs prediction-table, PT, to predict exactly whether a primary is executable. Simulation results show that DPA gets more execution time for primary and less wasted processor time compared to the well-known similar algorithms so far. The algorithm is being used in a RT-Linux based system and has realized faulttolerant function well.

\section{References}

1. Han C.C, Shin K.G, Wu J: A Fault-Tolerant Scheduling Algorithm for Real-Time Periodic Tasks with Possible Software Faults. IEEE Transactions on computers, Vol. 52 (2003) 362372

2. Han J.J, Li Q.H, Abbas A. Essa: A dynamic Real-Time Scheduling Algorithm with Software Fault-Tolerance. Journal of Computer Research and Development, Vol. 42 (2005) 315-321

3. Li QH, Han J.J, Essa A.A., Zhang W.: Dynamic scheduling algorithms with software faulttolerance in hard real-time systems. Journal of Software, Vol. 16 (2005) 101-107 